\title{
AN EXCEEDINGLY SHORT PROOF THAT THE HARMONIC SERIES DIVERGES
}

\author{
RICHARD L. BAKER
}

\begin{abstract}
In this short note we give an exceedingly short proof that the harmonic series diverges. The proof is virtually a one-liner.
\end{abstract}

\section{INTRODUCTION}

The harmonic series

$$
\sum_{n=1}^{\infty} \frac{1}{n}=1+\frac{1}{2}+\frac{1}{3}+\cdots
$$

is one of the most famous or well-known infinite series in elementary mathematical analysis. The series diverges - a fact first demonstrated by Nicole'd Oresme [1, ca. 1323-1382]. There are a number of proofs that the harmonic series diverges, some of them well-known and elementary. In [2], Steven J. Kifowit and Terra A. Stamps give a survey of 20 proofs of the divergence of the harmonic series, covering simple popular proofs, up to more advanced proofs.

\section{The Exceedingly Short Proof}

The author of the present note discovered the following elementary, almost one-line proof that the harmonic series diverges - this proof is not found in [2].

Theorem 2.1. The harmonic series diverges.

Proof. For all $x \geq 0, x \geq \ln (1+x)$. Therefore, we have the following exceedingly short proof that the harmonic series diverges.

$$
\sum_{n=1}^{\infty} \frac{1}{n} \geq \sum_{n=1}^{\infty} \ln \left(1+\frac{1}{n}\right)=\sum_{n=1}^{\infty}[\ln (n+1)-\ln n]=\lim _{n \rightarrow \infty} \ln (n+1)=\infty .
$$


R. L. BAKER

\section{REFERENCES}

[1] N. Oresme, Quastiones Super Geometriam Euclidis (Questions Concerning Euclid's geometry), (ca. 1360).

[2] S. J. Kifowit and T. A. Stamps, The Harmonic Series Diverges Again and Again, AMATYC Review, 27.2 (2006), 31-43.

MSC2010: 40A05

Key words and phrases: harmonic series, divergent series, real functions, summability, Oresme

Department of Mathematics, University of Iowa, Iowa City, IA 52242

E-mail address: richard-baker@uiowa.edu 